\title{
Capuchin Monkeys Alternate Play and Reward in a Dual Computerized Task
}

\author{
Audrey E. Parrish ${ }^{1 *}$, Sarah F. Brosnan ${ }^{1}$, and Michael J. Beran ${ }^{1}$ \\ ${ }^{1}$ Georgia State University \\ *Corresponding author (Email: audrey.parrish1@gmail.com)
}

Citation - Parrish, A. E., Brosnan, S. F., \& Beran, M. (2015). Capuchin monkeys alternate play and reward in a dual computerized task. Animal Behavior and Cognition, 2(4), 334-347. doi: 10.12966/abc.11.03.2015

\begin{abstract}
Many animals cooperate with one another to achieve outcomes that they could not obtain on their own. One form of cooperation that has received much attention but little empirical support from experimental studies is reciprocity, leaving open the question of whether animals will work to provide benefits to others. Although there is some evidence of reciprocal behavior in animals, early work in macaque monkeys showed that while they would initially work to pay another individual (who could then return the favor), they ceased doing so over time. Here, we extended this investigation of reciprocity to capuchin monkeys (Cebus apella), a species known for cooperating and providing each other with rewards in other contexts. We investigated whether capuchin monkeys would engage in a dual computer task that required alternating work by two animals, such that food rewards earned by one individual were delivered to the partner. Unlike previous research, capuchin monkeys sustained work on the task when their actions benefited only another monkey, even with progressively longer delays between the subject's actions that rewarded the partner and the partner's subsequent actions that rewarded the subject. A separate question was the degree to which subjects understood their actions as social. We assessed whether subjects' behaviors were influenced by the presence or absence of a partner. Subjects behaved differently in the two conditions, indicating that future research investigating what subjects understand about their partners is warranted.
\end{abstract}

Keywords - Capuchin monkey, Computerized testing, Social tasks, Delay tolerance, Inhibition

An important question in social cognition is the degree to which animals are willing to work together, a key component of social living. Many animals cooperate (e.g., Dugatkin, 1997), but cooperation varies substantially in the degree to which it requires investment by the partner. For instance, in mutualisms, animals work together for joint benefit, requiring neither investment nor any understanding of the nature of the task on which they are working (Brosnan, Salwiczek, \& Bshary, 2010; Brown, 1983). On the other hand, reciprocity requires individuals to invest now for a (potential) payoff in the future (Axelrod and Hamilton, 1981; although note that this may be accomplished without any understanding on the part of the actors). In some cases, reciprocity has explained costly cooperative behavior (e.g., Wilkinson, 1984). However, the degree to which reciprocity exists in nature, and therefore its importance for understanding the evolution of cooperation, is contentious, in part due to the difficulty researchers have had demonstrating reciprocal behavior in the lab (e.g., Brosnan et al., 2009; Melis, Hare, \& Tomasello, 2008; although see Dugatkin, 1991; Milinski, 1987; Rutte \& Taborsky, 2008).

Early laboratory attempts to determine whether monkeys would work to provide benefits to each other without simultaneously providing benefits to themselves provided only limited evidence of turntaking. An early series of studies assessed whether macaque monkeys would engage in a task in which their behavior resulted in the delivery of food to only a partner monkey (Boren, 1966; Colman, Liebold, \& Boren, 
1969). Initially, monkeys engaged in lever-pressing that delivered food to their partner. However, this behavior quickly extinguished if the actor monkey was not immediately rewarded for its own actions, even though joint effort by both monkeys would have led to nearly continuous delivery of food. In fact, responding decreased to such a low level that the study had to be stopped to avoid serious malnutrition for both individuals. The results suggested a fairly strong intolerance for sustained effort on a task in which the subject received no rewards while the partner did, even over very long trials that resulted in long delays between the subjects' action and receipt of food.

More recent studies, however, have demonstrated that other species of monkeys might be more likely to succeed in this sort of task. In particular, capuchin monkeys, a New World monkey genus known for high levels of cooperation in the wild (Crofoot, Rubenstein, Maiya, \& Berger-Wolf, 2011; Gros-Luis, Perry, \& Manson, 2003; Perry, Manson, Dower, \& Wikberg, 2003; Perry \& Rose, 1994) and in captivity (reviewed in Brosnan, 2010) provide a promising avenue for further research ${ }^{1}$. Aside from mutualistic cooperation, in laboratory tasks female brown capuchin monkeys show reciprocity in food sharing, sharing food more often with those who shared food with them previously (de Waal, 2000; de Waal, Luttrell, \& Canfield, 1993), and wild brown capuchin females showed the same reciprocal behavior with respect to grooming (di Bitetti, 1997; there is also more limited evidence of time-matched grooming in wild white faced capuchins; Manson, Navarrete, Silk, \& Perry, 2004). Brown capuchins also are more likely to share food with partners who help them in a cooperative task, but only if the partner's help is required (de Waal $\&$ Berger, 2000), and they may even engage in generalized reciprocity (Leimgruber et al., 2014). However, whereas capuchins are reciprocal, a recent study found no evidence for contingency (Suchak \& de Waal, 2012), indicating that reciprocity may be based on the symmetrical aspects of the relationship (e.g., symmetry-based reciprocity; Brosnan \& de Waal, 2002).

In the current study, we extended research with capuchin monkeys to a protocol more similar to the earlier reward-delivered-to-partner studies (Boren, 1966; Colman, et al., 1969). We investigated whether brown capuchin monkeys would engage in a dual computer task that required alternating work by two animals, such that food rewards earned by one individual were delivered only to a partner. Additionally, we included progressively longer bouts of effort that served only to deliver rewards to a partner to explore the temporal limits of such turn taking with capuchin monkeys.

Using a computerized task offered several benefits over previous work. First, all computerized testing requires some degree of tolerance for delayed rewards (i.e., animals respond and then food or other rewards are given elsewhere, such as from a dispenser). This aspect of stimulus-response-outcome spatial discontiguity might lend itself to success in the current study. Second, computerized tasks allow for better control over some aspects of testing than do manual tasks. They potentially eliminate problematic cues (e.g., the presence of the experimenter), and they typically allow for higher trial counts per testing session. Third, computerized tasks allow for control conditions that vary procedurally only on the presence or absence of the partner, something that is challenging to enact in manual tasks. Finally, a substantial portion of the existing literature on cognition involves computerized tasks, which is especially the case among capuchin monkeys (e.g., Beran \& Parrish, 2012; Beran \& Smith, 2011; Evans, Beran, Chan, Klein, \& Menzel, 2008; Fujita, 2009; Wright, 1999). Therefore, computerizing social cognition tasks allows for more comparability across cognition studies more broadly. We think that this is key for understanding the degree to which social aspects of the task influence cognition, and how the same underlying mechanisms influence "social" versus "physical" cognition.

An early study investigated joint computer play by examining the effects of competition on task performance in rhesus monkeys (Macaca mulatta) using a dual computer task that involved digitally 'shooting' at moving targets (Washburn, Hopkins, \& Rumbaugh, 1990). A speed-accuracy trade-off was observed in the competitive condition with monkeys hitting the target in significantly less time but using more shots in partner-present situations. These results suggested that the monkeys altered performance

\footnotetext{
${ }^{1}$ Note that to date the vast majority of fieldwork in the genus Cebus has been done with white faced capuchins, Cebus capucinus, whereas all of the lab work has involved brown capuchins, Cebus apella. Recently the argument has been made that these species should be in different genuses, with brown capuchins moved to the newly-created genus Sapajus (Alfaro et al., 2012); however, behaviorally the species are similar (Fragaszy et al., 2004) therefore for this study we consider them together.
} 
depending upon the social nature of the task. More recently, we have begun to investigate questions of economic decision-making across the primates using dual computer tasks. Rhesus monkeys, capuchin monkeys, and humans were tested on the Assurance Game using a two-player computerized version of the task in which participants had to coordinate their actions to maximize rewards (Brosnan, Wilson, \& Beran, 2012; Parrish, Brosnan, Wilson, \& Beran, 2014). The dual computer testing system we developed for this project allowed two participants to jointly respond to the task so that each partner's outcome was directly contingent upon the other's response. Critically, capuchin monkeys' choices were the same on the computerized and manual versions of the task, demonstrating that, whatever the monkeys actually understood about the task, their behavior across these two testing modalities was the same (Brosnan et al., 2011, 2012). This provides evidence that the monkeys responded to the computerized tests of social behavior similarly to the more common manual tests (e.g., bar pull tasks, exchanges). The advantage to the computerized format was that it allowed us to create a truly "functionally synchronous" version of the game in which there was no way for the players to see each other's choices prior to making their own choice, something we could not do with the manual task, and then to compare these responses to those in the otherwise-identical version of the task in which they could see their partner's choices. Here, we extended this methodology to assess the degree to which primates were willing to engage a turn-taking task in which food rewards earned by one individual within a pair were delivered only to the second individual.

We also introduced a partner-absent control in which solitary monkeys played against a simulated partner whose actions replicated those produced by the monkey's real partner in a previous test session. This partner-absent control condition is directly tied to our second rationale for using a computerized procedure. Thus far, we have focused on whether a behavior functions as cooperation; in other words, do both individuals benefit? This approach, which focuses on costs and benefits, makes no assumptions about what, if anything, the animal understands about the task. This functional approach is the dominant one in evolutionary biology (e.g., Bshary \& Bergmuller, 2008) and is distinct from the more mechanisticallyfocused psychological approach. We are taking an explicitly functional approach here as well, but we are nonetheless interested in how the social context may be influencing the monkeys' decision-making. This is important both theoretically, to see how social context influences decision-making, and also methodologically, to see how jointly performed tasks are influenced by the presence of a social partner.

"Social context" has a range of meanings. At the most cognitively demanding, it implies that the animals understand that they are helping their partner. This is important, but we first began with the most basic question; did the monkeys' behavior change for the same task when the partner was present versus absent? We achieved this by yoking the partner-absent sessions to the partner-present sessions such that the computer exactly replicated the real partner's previous play, including delay periods to the reward. The only procedural differences between the partner-present and partner-absent condition sessions were (1) whether a partner monkey was present and (2) any changes in the subject's response times (which we could not control). If the monkeys' behavior was different, this would provide evidence that social context was influencing behavior in this task. Although this does not tell us anything about the monkeys' understanding of their partner's role, it does tell us whether they respond to the difference in social context (partner-present versus partner-absent), and this is a critical first step that must be addressed prior to asking further questions about their understanding of their and their social partner's roles.

For Experiment 1, we developed a protocol for implementing a turn-taking procedure in which monkeys would participate, with the goal of having them tolerate long delays between when they responded (to reward a partner) and when they were rewarded in return. Past research suggested this would be difficult to accomplish (Boren, 1966; Colman et al., 1969), but the nature of computerized testing suggested it was possible. Once we established this, for Experiment 2, we wanted to determine whether the monkeys could maintain this turn-taking behavior. Again, despite previous research with macaque monkeys, we hypothesized that if capuchin monkeys learned the contingencies of the task, they would maintain the behavior in order to maximize their rewards. Finally, we assessed whether there were changes in performance when monkeys performed the task with a monkey partner or alone to determine whether the social context influenced their behavior. We did not have a directional hypothesis, however if we found 
changes in behavior, this would indicate the need for future experiments to determine how they perceived their social partner's role in the task.

\section{Experiment 1 - Training Joint Computerized Testing}

\section{Methods}

Subjects. We tested 10 capuchin monkeys (Cebus apella), six males (ages 4 to 21 years) and four females (ages 8 to 15 years). All monkeys lived in multi-male, multi-female social groups, including offspring, in indoor/outdoor enclosures with extensive enrichment available. Monkeys had served as test subjects in a number of previous computerized tests (e.g., Beran, 2008; Beran \& Parrish, 2012; Beran \& Smith, 2011), including a study involving a joint computer screen with two players (Brosnan et al., 2012). Monkeys were rewarded for certain responses to the apparatus with $45 \mathrm{mg}$ fruit-flavored food pellets, and they had continuous access to water. They received a daily diet of chow, fruits and vegetables, and they were never food deprived or weight reduced.

Materials. Testing was conducted using the Language Research Center's Computerized Test System that is comprised of a computer, digital joystick, color monitor, and pellet dispenser (see Evans et al., 2008, for details on training). Monkeys manipulated the joystick to produce isomorphic movements of a computer-graphic cursor on the screen, and pellets were delivered using a dispenser interfaced to the computer through a digital I/O board (PDISO8A; Keithley Instruments, Cleveland, OH).

Turn-taking task. The task required two monkeys to alternate turns by responding one at a time to a multi-player computerized task in which the rewards that each animal earned were delivered to a partner. That is, if Monkey A completed a trial, Monkey B was then rewarded (Monkey A got no rewards), following which Monkey B had the opportunity to complete a trial for which Monkey A would receive the rewards. This differs from a traditional delayed reward paradigm in that rewards were not guaranteed contingent on responses by a given player because the partner could choose not to participate (or choose to complete trials at a slower pace than the other monkey had done previously). These contingencies meant variable - and possibly longer - delays between action and subsequent reward that were not contingent upon the player's own behavior.

Procedure. For joint-testing, monkeys were separated as pairs into adjacent testing boxes from which they could clearly see each other completing the task and eating any food rewards. To emphasize that the monkeys were engaged with a partner, monkeys shared a single computer monitor (see Figure 1). This is a critical change to the typical computerized testing procedure as it may emphasize to the monkeys the joint nature of the task. Each individual had its own joystick controller and pellet dispenser, making it impossible to interfere with each other's turn or to take each other's rewards. Monkeys played a psychomotor game called CHASE that involved tracking moving targets on the computer screen and contacting them with a cursor controlled by isomorphic movements of the joystick. The monkeys already were familiar with the CHASE task (Evans et al., 2008), but in the context of this experiment, there were a number of novel variations on the contingencies for performing the task and on the outcome for completing trials. Monkeys progressed through a series of training conditions designed to assure that they understood the contingencies of the task and would tolerate the turn-taking protocol.

Training. There were six training conditions, each at a higher level of complexity than the previous condition. See below for a complete description of each training condition, including criterion and session counts for each pair of monkeys. These criteria were established a priori, although in the Results section it will be evident that in some cases we had to modify the criteria when monkeys' performances indicated that they were not capable of meeting those criteria. Developing this training routine was one goal of this project, and so we wanted to have specific criteria in place at the outset, although we anticipated that those 
might need to be modified.

Training condition 1. Condition 1 was the simplest task in which the monkeys each worked on their own half of the screen at the same time for their own rewards to familiarize them with working on this task on a shared computer monitor. The computer screen was split with a vertical dividing line pixelated onscreen so that one animal could move only its cursor on one half of the screen, and the other animal on the other half (spatially matched to their relative left-right locations within their two test boxes). Here, there was no need for coordination of any type, and monkeys were given food pellets for tracking and contacting all three large moving target stimuli on their half of the screen on each trial. These targets moved in a straight line in a randomly determined direction on the screen, and then "deflected" off the edges of the screen or vertical barrier in a new direction whenever they hit an edge. Once they completed a trial, they were presented with the next trial, independent of the actions of the partner monkey during that time. A pair of monkeys would progress when they had completed a single session in which both members of the pair completed at least 200 trials.

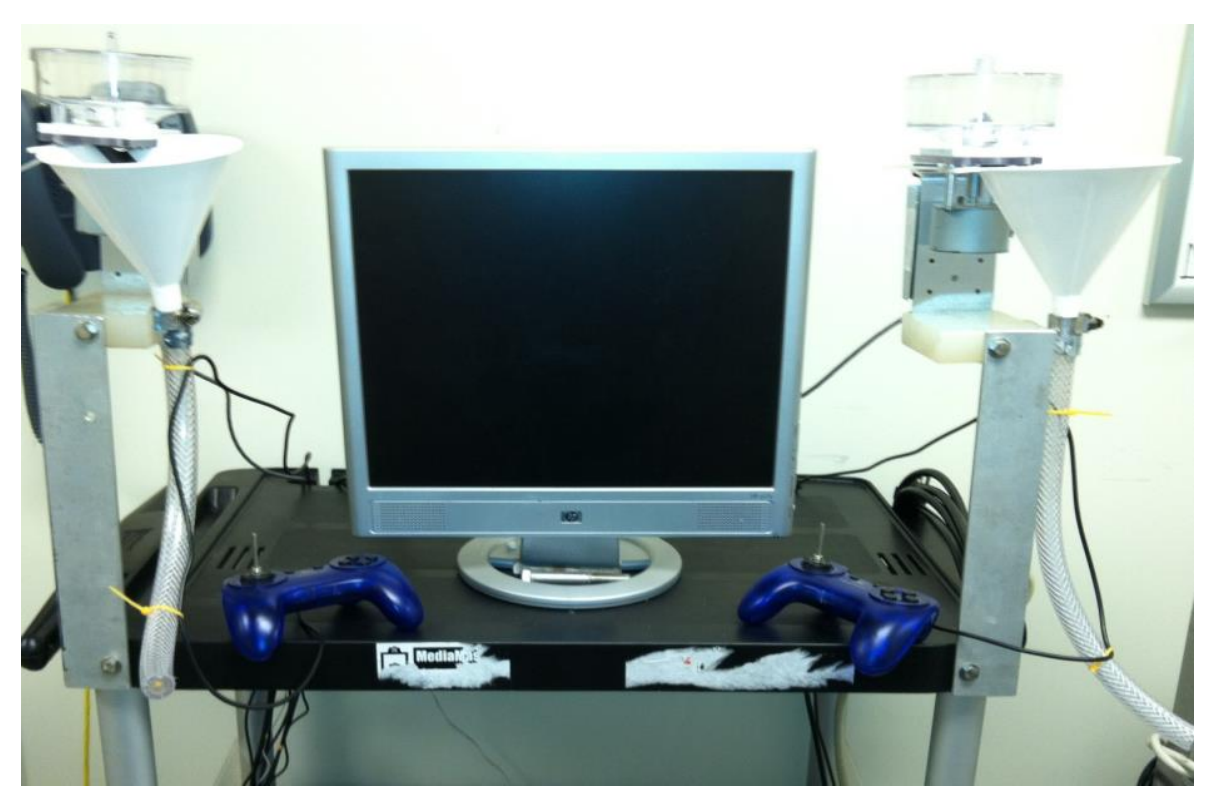

Figure 1. Joint computerized apparatus used for testing. Pairs of monkeys shared one computer screen but had their own joystick that would attach to their testing box and pellet dispenser.

Training condition 2. Condition 2 introduced the first requirement for coordinated action by the monkeys. The task alternated so that one monkey at a time had to work for its own reward, but now the partner had to inhibit touching the joystick when it was not their turn. Otherwise, the program would freeze until the partner released the joystick. The screen looked exactly the same as in Condition 1, but now targets appeared on only one half of the screen on each trial. The monkey who controlled the cursor on that half of the screen could contact those targets and, when all were cleared, obtain a food reward. The monkey who controlled the cursor on the other half of the screen had to inhibit deflecting the joystick in any direction because any movement of that joystick would freeze both cursors. A pair of monkeys continued in this condition until they completed a session with at least 150 completed trials per player, at which point they moved to Condition 3. If they completed three consecutive sessions of Condition 2 without doing at least 50 trials in each of those sessions, they were moved back to Condition 1 to be retrained.

Training condition 3. In this condition, the digital divider on the screen was removed so that both players' cursors had access to the entire screen and rewards were delivered to both monkeys rather than just the playing monkey. The cursor color that matched the color of the target stimuli indicated which player was allowed to move the joystick (and control its cursor). Successful completion of a trial led to food delivery to both animals. This version of the task involved the need for inhibition by the non-playing 
partner; again, if the non-playing partner failed to inhibit, the cursor froze. The non-matching cursor color indicated which monkey needed to inhibit interacting with its joystick. Monkeys moved to Condition 4 after a session in which they completed at least 150 total trials between them. However, if they completed three consecutive sessions with fewer than 80 completed trials total, they were moved back to Condition 2 for additional training.

Training condition 4. The new manipulation in Condition 4 was that the playing monkey's actions led to food delivery to its partner only. Initially, monkeys completed only a single trial before it was their partner's turn. When they completed a session in which they successfully alternated between players, we increased the number of consecutive trials required of each player to three before it became the partner's turn. When monkeys successfully alternated playing at 3-trial blocks, they were moved to 5-trial blocks. Monkeys continued in this 5-trial condition until they completed three consecutive sessions of at least 150 trials, indicating that they could sustain this pattern of alternated play.

Training condition 5. Condition 5 instituted a number of design changes. First, we implemented a titration procedure that progressively increased the number of trials that the player monkey had to complete before roles were switched between player monkey and partner monkey. The program titrated the number of trials in the block, so that at the outset each player completed one trial in alternation, and then two trials per player, and so forth throughout each test session. Second, only two target stimuli appeared on each trial instead of three. Note that the non-playing partner still had to inhibit any movement of the joystick or else the playing partner's cursor froze on the screen. This was the most difficult test the monkeys faced in this experiment because it required both the inhibition component and included the titration procedure, the latter of which led to trial blocks that could be quite long, with all rewards being delivered only to the non-playing partner. Monkeys did not perform well in this condition - they produced very few trials and often refused to engage in the task (see Results for quantitative information).

Training condition 6. Condition 6 was the final training condition and was identical to Condition 5 except that we removed the requirement that partner monkeys not touch the joystick while the player monkey was working (e.g., we removed the inhibition requirement).

\section{Results}

Table 1 provides the total number of sessions each monkey completed in each training condition. The pairs that were tested initially were started in Condition 3, before we realized the need for more remedial training conditions, and later pairs began in Condition 1, as indicated in the table. Not all pairs met criterion in all training conditions, primarily due to the inhibition component with the joystick when it was not a player's turn to act, a requirement that was too difficult for most pairs to sustain. Thus, even though we had established training criteria a priori, the training conditions that were required in actuality by different pairs varied as we learned how to instill joint game playing by monkeys in this experimental paradigm. Once we had established that monkeys would return to engaging the task more extensively without the inhibition component, they were moved to Experiment 2.

Table 1

Total Number of Sessions Completed in each Training Condition for all Monkey Pairs

\begin{tabular}{cccccc}
\hline $\begin{array}{c}\text { Training } \\
\text { Condition }\end{array}$ & Drella-Wren & Gabe-Nala & Griffin-Lily & Nkima-Gambit & Liam-Logan \\
\hline 1 & 2 & $* 5$ & 2 & -- & $*-$ \\
2 & 6 & 3 & 5 & $* 13$ & 1 \\
3 & $* 15$ & -- & 5 & 8 & 2 \\
4 & -- & -- & 5 & 5 & 6 \\
5 & -- & -- & 4 & 3 \\
6 & 1 & 2 & 2 & 45 \\
\hline
\end{tabular}

Note. Asterisks indicate the training condition that each pair started on. For the first four pairs, the first animal name indicates the male monkey. The Liam-Logan pair consisted of two males. 


\section{Discussion}

The training protocol was successful in establishing turn-taking behavior among the monkeys, but only when we removed the requirement of total disengagement of the joystick by the non-playing partner. This constant engagement of the joystick might suggest that the monkeys did not really come to see themselves as being in either the "player" role or the "partner" role. This would be a problem for concluding that the monkeys recognized the task as involving themselves and another individual. However, it did not appear that both monkeys constantly used the joystick during trials, but rather that the non-playing partner would grab and test the joystick occasionally, perhaps to determine whether it was its turn to play. Due to this observation, we moved the monkeys to Experiment 2 in which we could assess willingness to engage in the turn-taking procedure under more controlled conditions. We also implemented a measurement of how much time the partner engaged the joystick when it was not playing, so that we could quantify this degree of behavioral interaction and also better understand how the monkeys viewed the nature of the task.

\section{Experiment 2}

\section{Method}

Subjects and apparatus. These were the same as in Experiment 1.

Procedure. Each monkey had a cursor that appeared in a randomized location on the computer screen. The cursors differed in color (pink or green) so that, with movements of the joystick, the monkeys could discern which cursor they controlled, and those cursors moved in directions isomorphic to the movements of the joysticks controlling each cursor. At the same time that a cursor appeared, the monkeys also saw two larger colored (pink or green) circles appear on the computer screen. Touching a target with the cursor of the other color removed that target from the screen. When all targets were removed, only the non-playing partner received food pellets. At the start of each session, we randomly determined which monkey acted as the first or second player so that monkeys could not simply associate a particular cursor/target with their turn throughout the whole experiment (although they could do so within a single session). This ensured that, session by session, they had to use cues about cursor movement and their own manipulation of the joystick on the earliest trials to recognize which cursor they operated for that session.

We made a number of design decisions to indicate to the monkeys in as many ways as possible who was working and who would be rewarded. First, the color of the targets always indicated which of the two monkeys would get pellets at the end of the trial. The targets were always opposite in color to the cursor that could remove them. Thus, when the player controlling the green cursor removed the pink targets from the screen, the non-playing partner received two food pellets for that trial (despite not having to do any work, or contact any targets). Second, the screen changed background color as another indicator of which cursor was active so that across sessions the background color always indicated which cursor was active, independent of which monkey was in control of that colored cursor in that session. The pink cursor was always associated with a yellow background, whereas the green cursor was always associated with a white background. Third, the non-playing monkey's cursor was frozen on the screen. Throughout the session, the playing monkey had to complete the trial in order for that trial to end. There was no programmed termination of a trial based on the end of a delay interval, and there was nothing that the non-playing partner could do to progress the task to the next trial. In this way, for subjects to earn rewards they had to work for rewards that always went to the partner so that their partner then could work for rewards that always went to them. It is important to note that a playing monkey whose rewards were being delivered to its partner not only had to work while the partner received rewards, but also had to wait until its partner completed its own trials before receiving any food. Therefore, by definition, this task required turn taking as the monkeys only got fed by the actions of a partner, who received nothing in return for that effort.

Within a session, we titrated the number of consecutive trials that a monkey had to complete before the targets changed color and the players switched roles. At the outset, each monkey had to perform just 
one trial and then turns switched. This occurred for five alternations across the partners, and then each player had to complete two consecutive trials before their partner got a turn, and so on throughout the duration of the 120-min session. Each pair of monkeys completed five sessions in this phase of the experiment.

Solo condition. Next, the monkeys each completed five solo sessions. In these sessions, a monkey worked alone, without another monkey in the adjacent box. The task for the playing monkey was similar to the experimental condition, except that now the program simulated the role of the partner in terms of the time delay to completing trials (these delays were actual delays generated by the real partner on a previous social condition session). On its turn, the monkey still had to track and contact targets in the same way as in the experimental sessions. However, when the targets changed color to those that would have been completed by the (now absent) partner, the computer simulated a delay period before dispensing pellets to the real monkey (as if the trial had been completed; note that pellets that were earned by the responses of the ghost monkey were dispensed to a tube attached to the partner's pellet dispenser). The solo player's cursor never moved on the screen during the ghost player's turn.

These solo delay periods were yoked to true delay periods produced by the (now absent) partner for that monkey from the earlier five sessions (e.g., social session 1 was yoked to solo control session 1, etc.). In this way, we matched how long the focal monkey subject had to wait to get pellets when it was not its turn to play, such that any differences in the subjects' performance with or without a partner monkey present could not be the result of differences in the speed of responding between the real partner and the "ghost." Thus, the only differences between the social and solo condition sessions were (1) whether a partner monkey was present, (2) whether a cursor moved and contacted targets when the real monkey was in the role as the partner, and (3) any changes in the subject's response times.

\section{Results}

For test and solo conditions, we first verified that the data (mean number of trials completed) were normally distributed, and this was true for both conditions (solo condition: Kolmogorov-Smirnov test, $D(10)=0.16, p=0.20$; partner-present: $D(10)=0.23, p=0.16)$.

Monkeys were successful in sustaining performance in this task. By the end of each session, monkeys were working for long periods of time while receiving no direct food rewards for their own efforts. By the last block of trials for test sessions, monkeys were working an average of $129.28 \mathrm{~s}$, or over two minutes, to complete their runs of trials while the partner received the rewards (see individual data in Table 2). In some cases, monkeys were doing 10 or more trials in a row without any reward, during which time their partner was receiving 20 or more pellets, which took upwards of four minutes. Thus, at the qualitative level, our task did promote extended working by monkeys that received no immediate direct benefit for that work.

Despite the removal of the required joystick-inhibition component in Experiment 2, partner monkeys largely inhibited use of the joystick when it was not their turn. The average duration of partner joystick deflection $(M=3.50 \mathrm{~s}, S E=0.44)$ was significantly lower than the average trial duration for the playing monkey $(M=10.92 \mathrm{~s}, S E=0.62 ; t(9)=-11.15, p<0.001)$, a measure which reflected ongoing joystick deflection by the playing monkey. Another measure of joystick-inhibition by the partner monkey involved tallying discrete deflections of the joystick when their cursor is not active. On average, partner monkeys engaged in only 2.90 total joystick deflections per trial. We also examined the proportion of trials in which the non-playing partner never deflected the joystick. This would be the purest indication that they did not attempt to engage in the task. Table 3 shows individual results for the joystick inhibition data, including the proportion of trials for each monkey in which they never deflected the joystick when it was not their turn. 
Table 2

Individual Data for the Average Time on Task for the Last Block of Trials (in seconds) across the Five Sessions of the Social Condition for each Pair of Monkeys

\begin{tabular}{|c|c|c|}
\hline & Monkey Players & $\begin{array}{c}\text { Average time on task for } \\
\text { the last block of trials (in seconds) }\end{array}$ \\
\hline \multirow[t]{2}{*}{ Pair 1} & Drella (Male) & 150.11 \\
\hline & Wren (Female) & 139.90 \\
\hline \multirow[t]{2}{*}{ Pair 2} & Gabe (Male) & 225.60 \\
\hline & Nala (Female) & 130.55 \\
\hline \multirow[t]{2}{*}{ Pair 3} & Griffin (Male) & 164.17 \\
\hline & Lily (Female) & 118.00 \\
\hline \multirow[t]{2}{*}{ Pair 4} & Nkima (Male) & 100.49 \\
\hline & Gambit (Female) & 100.17 \\
\hline \multirow[t]{2}{*}{ Pair 5} & Liam (Male) & 90.18 \\
\hline & Logan (Male) & 73.66 \\
\hline
\end{tabular}

Table 3

Joystick Deflection Data for each Monkey Across all Partnered Sessions

\begin{tabular}{ccccc} 
Monkey as Partner & $\begin{array}{c}\text { Average } \\
\text { Number of } \\
\text { Deflections Per } \\
\text { Trial }\end{array}$ & $\begin{array}{c}\text { Average Joystick } \\
\text { Deflection Duration } \\
\text { (in seconds) }\end{array}$ & $\begin{array}{c}\text { Average Trial Length of } \\
\text { Playing-Monkey } \\
\text { (in seconds) }\end{array}$ & $\begin{array}{c}\text { Proportion of Trials } \\
\text { with No Joystick } \\
\text { Deflection by this } \\
\text { Partner Monkey }\end{array}$ \\
\hline Drella (Male) & 4.17 & 4.52 & 10.45 & 17.20 \\
Wren (Female) & 6.41 & 5.16 & 12.94 & 5.97 \\
Gabe (Male) & 2.28 & 3.84 & 13.63 & 31.75 \\
Nala (Female) & 2.85 & 4.94 & 11.89 & 25.29 \\
Griffin (Male) & 3.36 & 3.99 & 10.20 & 22.90 \\
Lily (Female) & 1.65 & 3.67 & 9.14 & 46.25 \\
Nkima (Male) & 0.38 & 0.83 & 12.91 & 74.87 \\
Gambit (Female) & 6.07 & 3.87 & 11.44 & 55.45 \\
Liam (Male) & 1.25 & 2.32 & 7.57 & 51.39 \\
Logan (Male) & 0.62 & 1.83 & 9.07 & 72.82 \\
\hline
\end{tabular}

During initial data analysis, we noticed an intriguing difference in male and female performance in the solo condition. Thus, as a post hoc analysis, we examined the effect of sex/dominance (note that sex and dominance were confounded given that the male monkeys were dominant to the female monkeys in all male/female pairs) and condition on the number of trials completed by the monkeys using a mixed-design, repeated-measures ANOVA with sex/dominance as a between-subjects factor and condition as a withinsubjects factor. When we separated the pairs by sex/dominance, we found no main effect of condition (e.g., partner present vs. solo control: mixed ANOVA: $F(1,6)=0.53, p=0.49)$ or sex/dominance $(F(1,6)=4.15$, $p=0.09)$, but there was an interaction between sex/dominance and condition $(F(1,6)=20.73, p=0.004$; $\eta \mathrm{p}^{2}=0.78$ ). Males and females differed in terms of the effect of condition on performance (see Figure 2 ). Males completed significantly fewer trials in the solo condition compared to when tested with their female monkey partner $(t(3)=4.24, p=0.02)$. Although the results for female monkeys did not reach statistical significance, there was a trend for females to complete more trials when tested in the solo conditions compared to when tested with their male monkey partner $(t(3)=-2.96, p=0.06)$. Note that we did have one male-male pair (all others were mixed-sex). For this pair (Logan-Liam), their dominance rank was unclear as they were related (sibling) sub-adult males. They were not included in this analysis.

To determine whether these changes in performance when shifting from the partner condition to the solo condition were driven by differences in the number of trials completed for only the first or second session in the solo condition, we calculated a correlation to assess whether males or females significantly increased or decreased the number of trials completed across the solo condition sessions. We found no correlation between session number and the number of trials completed for either sex (males: $r(18)=0.03$, $p=0.90$; females: $r(18)=0.09, p=0.71$ ), indicating that performance was consistent across all solo 
condition sessions for both sexes. Thus, the introduction of the solo condition did not lead to an immediate but short-lived change in the monkeys' behavior, but rather a sustained change throughout the entire solo condition that depended on the sex/dominance of the individuals being tested in the solo condition. There was also no correlation between the number of trials the pairs completed and session number across the partner-present sessions (males: $r(18)=0.13, p=0.59$; females: $r(18)=0.13, p=0.59$ ). Together, these results indicate that partner presence or absence had an effect on the performance of subject monkeys in this dual computer task.

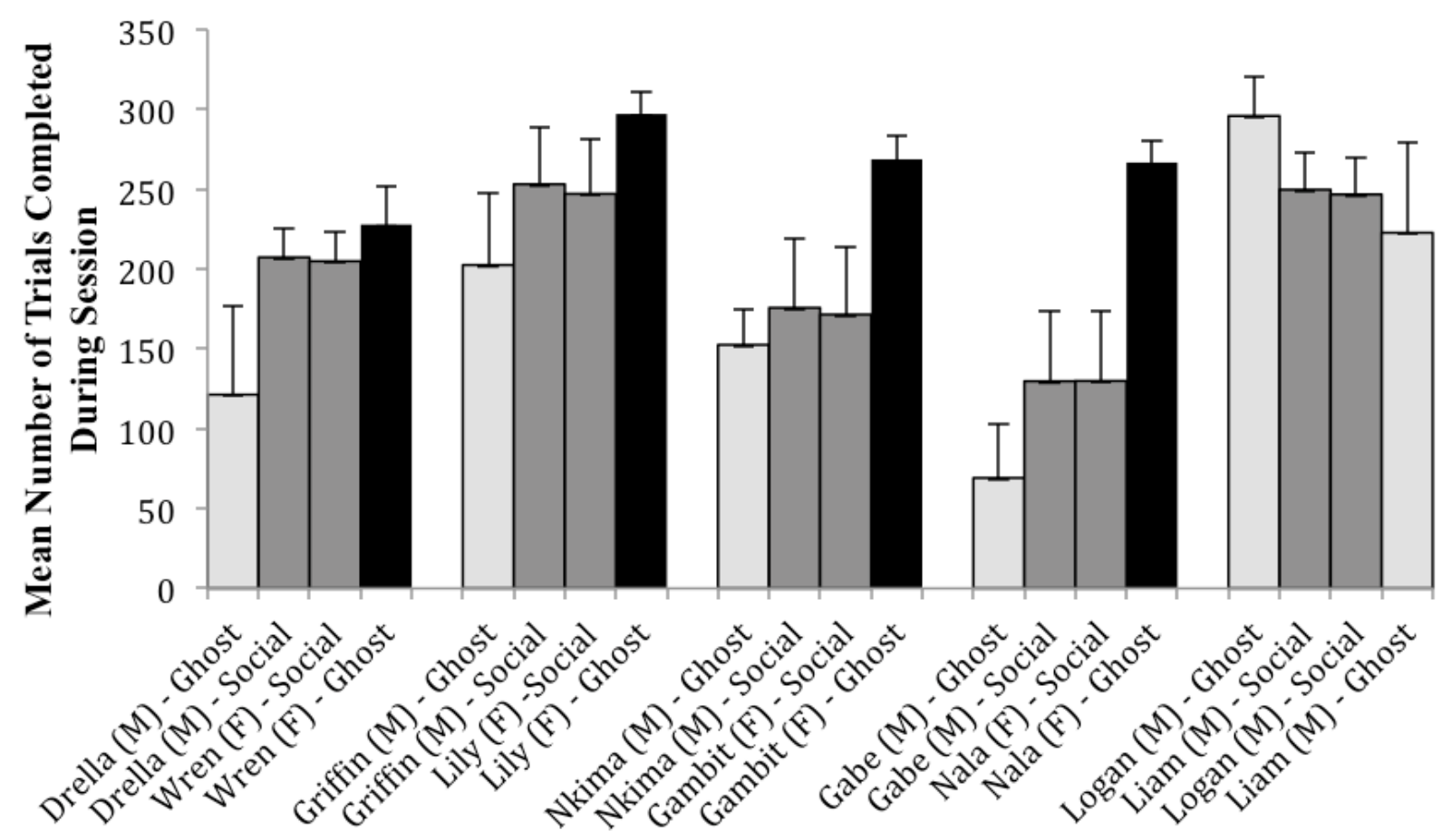

Figure 2. Mean number of trials completed by each pair of monkeys in the social condition (when paired with their partner monkey), and for each individual when tested in the ghost condition (no partner present). Dark grey bars show performance as a pair. Note that these two values will be slightly different because one animal could do a few more trials than the other depending on when the session ended. Light grey bars are male monkeys tested alone, and black bars are female monkeys tested alone. The error bars indicate standard errors of the mean.

\section{General Discussion}

Our results indicated that capuchin monkeys were capable of engaging in reciprocal behavior, as defined by effort expended for gains that only went to another individual. Monkeys sustained work in this dual computer turn-taking task in which their immediate actions benefited only another monkey for hundreds of trials within a session, while withstanding progressively longer delays between their actions that rewarded the partner and the partner's subsequent actions that rewarded them. In some cases, subjects were waiting nearly four minutes (with an average of more than two minutes) between completing a trial and receiving a reward after their partner subsequently completed its trials. In addition, partner monkeys largely inhibited joystick use when it was not their turn, suggesting that they understood when they could and could not participate in the task. Beyond the overall group success at the task, we found some evidence that the social context of the task influenced their behavior, which indicates that it is worthwhile for future studies to explore what the monkeys understand about their social partner's role in the task.

Sustaining performance on any task in the face of such lengthy and uncertain delays to rewards is impressive. In a traditional choice task with delayed rewards, some capuchin monkeys have shown the 
capacity to wait approximately 80 seconds for a delayed outcome (e.g., Addessi, Paglieri, \& Foracolo, 2011). However, in many cases monkeys will not choose rewards that are delayed by more than a few seconds over those delivered more immediately (Ramseyer Pelé, Dufour, Chauvin, \& Thierry, 2006). The sometimes impulsive nature of capuchin monkeys (e.g., Evans, Beran, Paglieri, \& Addessi, 2012) could be expected to work against successful performance of a task that requires prolonged effort that subsequently does not deliver a reward to the player, but rather to another individual. This is likely one reason that it has been difficult to demonstrate sustained turn-taking behavior in laboratory tests with nonhuman species (e.g., Boren, 1966; Colman, et al., 1969).

Moreover, the use of a solo condition, in which the computer instantiated time delays yoked to those given by the real partner in an earlier social condition, demonstrated that the presence of a partner influenced subjects' performance. Male capuchin monkeys completed significantly fewer trials when tested in the solo condition (alone) than in the social condition (paired with a female), but female capuchin monkeys completed more trials when tested in the solo condition (alone) than in the social condition (paired with a male). This result cannot be explained by methodological issues relating to the computer task itself. The reward schedule and "partner" choice timing were identical between the partner and solo conditions. In both conditions, pellets were dispensed to the non-playing partner (or the empty cage), and all other task parameters were the same for both sexes. If this were a temporally or experientially driven effect, we would anticipate that males and females would change their behavior in the same direction.

We are unsure whether the deviation is due to the individuals' sex or their rank, as dominance and sex were confounded in all four mixed-sex pairs (as is typical for capuchins, all of our male capuchins outranked their female partners), and the one male-male pair (Liam-Logan) was uninformative because it involved two related animals with a very close and fluctuating dominance relationship, and whose performance was relatively stable across conditions. We propose that a sex difference is the more likely candidate (over dominance) for several reasons. Male capuchin monkeys are more indiscriminate in their food sharing and grooming, whereas female capuchin monkeys are more contingent, sharing with and grooming only those who share with or groom them (de Waal, 1997; Di Bitetti, 1997). Moreover, it is possible that females were more food motivated in the current task, something that has been reported in other New World monkey species (e.g., Callithrix jacchus, Michels, 1998), although we find this possibility highly unlikely given that these monkeys are not food deprived for testing purposes nor have shown this sex difference in other non-social computerized tasks.

It is possible that the females were more efficient when working the task alone due to lower stress levels without a more dominant male partner present. Primates sometimes show rank-related social modulation of performance in which subordinate monkeys voluntarily inhibit responding to a known task in the presence of dominant individuals (e.g., Drea \& Wallen, 1999). Males may have worked more efficiently when a female partner was in close proximity to them, allowing them to focus on the task rather than attending to where that female was in relation to other group members. Given the small number of subjects and the post hoc nature of the results, it is premature to draw any firm conclusions, but this pattern of results shows that the monkeys, at minimum, responded differently when playing the game with another monkey present next to them as compared to playing it without a partner monkey being present. Future research is needed to help determine what specific feature of the social context caused this deviation in behavior, and why it differed between males and females.

We also do not know why the monkeys' behavior changed; at the most basic it could be that they are more (or less) likely to work on any task in the presence of a conspecific as compared to when alone. Alternately, they may be more (or less) likely to work when benefitting a partner, but this will require future work as our task was not designed to answer this question. Because the task was difficult to train, we did not counterbalance the order of learning (solo vs paired), and due to the nature of the task, we would not expect the monkeys to change their behavior in the solo condition unless the social context affected them. The tasks were identical, and the only way that they could obtain rewards on either condition was by completing the task, so it actually is interesting that their behavior changed at all. In future work, we hope to explore whether the monkeys understand that they are helping a partner. This is a possibility; capuchins are known to make choices that benefit a partner (e.g., in prosocial tasks; Brosnan et al., 2010; Claidière et 
al., 2015; de Waal et al., 2008; Lakshminarayanan \& Santos, 2008). Therefore, one avenue for future testing is to explore whether monkeys choose an option that helps both themselves and a partner, as opposed to just themselves, and whether they are sufficiently sensitive to their role in helping their partner to prefer the "prosocial" option in a paired condition more often than in the solo condition (e.g., Jensen, Hare, Call, \& Tomasello, 2006; Silk et al., 2005).

The dual computerized testing paradigm that we employed here provides a methodology for assessing cooperative or coordinated behavior with control over multiple factors. With the current paradigm, we can yoke temporal delays between test and control conditions and hold more aspects of the procedure consistent across these conditions, allowing for good procedural control and the opportunity to create tightly-controlled solo conditions. However, there are critical aspects to consider when instantiating this methodology. Certainly, our training regime worked because we first allowed the monkeys to benefit themselves directly, and then we subsequently advanced them into a task where they paid only their partner and not themselves. We also found it useful to titrate the degree to which subjects played to pay only the partner from very short periods (e.g., a single trial) to much longer ones by session end.

We also learned that requiring total disengagement from the joystick by non-playing partners was difficult or even impossible to accomplish. This was initially a concern, because it suggested the monkeys were not viewing the task as one where sometimes they worked but sometimes they did not (but might still get paid); without this understanding, the monkeys might not recognize that they were participating in a delayed reward task. However, data from Experiment 2 indicated that non-playing monkeys only occasionally engaged the joystick when it was not their turn to play. These occasional engagements were likely for a good reason. Despite our attempts to provide what we thought were clear visual indicators of which monkey was the player, the monkeys themselves likely preferred the most direct way of determining that - moving the joystick and seeing if isomorphic movements occurred onscreen. If they did not, the monkeys released the joystick. Thus, given the challenges associated with complete disengagement from a familiar, positive stimulus (the joystick), the requirement for total inhibition of manipulation of the joystick is not a design aspect that will be easy to incorporate.

Between the present results and our earlier data showing continuity in decision-making among humans and nonhuman primates in a dual computer task (Brosnan et al., 2012), and similar outcomes in a computerized and manual version of the same coordination task (Brosnan et al., 2011, 2012), we have evidence that these two-player computer tasks have the potential to contribute to a fuller understanding of the nature of social cognition and decision-making in nonhuman animals. With proper training, the dual computer tasks can be introduced and modified to ask a number of questions that require two (or more) players to engage the same task. Whether cooperative or competitive in nature, such tasks can be designed to mimic in function the kinds of situations that involve joint engagements in real world contexts between social partners. Thus, they can be a valuable tool in animal behavioral research, and also could be easily adapted for use with adult humans and children to also allow for new avenues for comparative approaches to social cognition research.

\section{Acknowledgements}

The authors thank Ted Evans, Bonnie Perdue, Betty Chan, and the animal care staff at the Language Research for their assistance with data collection and for insightful comments on this project. This research was supported by grants from the National Science Foundation (SES 1123897, SES 0729244, and SES 0847351) and the National Institutes of Health (HD060563) to SFB and MJB, and a GSU Brains and Behavior Fellowship to AEP. 


\section{References}

Addessi, E., Paglieri, F., \& Focaroli, V. (2011). The ecological rationality of delay tolerance: Insights from capuchin monkeys. Cognition, 119, 142-147.

Alfaro, J. W. L., Silva, J. D. S. E., \& Rylands, A. B. (2012). How different are robust and gracile capuchin monkeys? An argument for the use of Sapajus and Cebus. American Journal of Primatology, 74, 273-286.

Axelrod, R., \& Hamilton, W. D. (1981). The evolution of cooperation. Science, 211, 1390-1396.

Bakeman, R. (1992). Understanding social science statistics: A spreadsheet approach. Hillsdale, NJ: Lawrence Erlbaum Associates, Inc.

Beran, M. J. (2008). Capuchin monkeys (Cebus apella) succeed in a test of quantity conservation. Animal Cognition, $11,109-116$.

Beran, M. J., \& Parrish, A. E. (2012). Sequential responding and planning in capuchin monkeys (Cebus apella). Animal Cognition, 15, 1085-1094.

Beran, M. J., \& Smith, J. D. (2011). Information seeking by rhesus monkeys (Macaca mulatta) and capuchin monkeys (Cebus apella). Cognition, 120, 90-105.

Boren, J. J. (1966). An experimental social relation between two monkeys. Journal of the Experimental Analysis of Behavior, 9, 691-700.

Brosnan, S. F. (2010). What do capuchin monkeys tell us about cooperation? In D. R. Forsyth. \& C. L. Hoyt. (Eds.), For the greater good of all: Perspectives on individualism, society, and leadership perspectives on individualism, society, and leadership (pp. 11 -28). New York: Palgrave Macmillan Publishers.

Brosnan, S. F., \& de Waal, F. B. M. (2002). A proximate perspective on reciprocal altruism. Human Nature, 13, 129152.

Brosnan, S. F., Houser, D., Leimgruber, K., Xiao, E., Chen, T., \& de Waal, F. B. M. (2010). Competing demands of prosociality and equity in monkeys. Evolution of Human Behavior, 31, 279-288.

Brosnan, S. F., Parrish, A., Beran, M. J., Flemming, T., Heimbauer, L., Talbot, C. F., ....Wilson, B. J. (2011). Responses to the assurance game in monkeys, apes, and humans using equivalent procedures. Proceedings of the National Academy of Science, 108, 3442-3447.

Brosnan, S. F., Salwiczek, L., \& Bshary, R. (2010). The interplay of cognition and cooperation. Philosophical Transactions of the Royal Society B: Biological Sciences, 365, 2699-2710.

Brosnan, S. F., Silk, J. B., Henrich, J., Mareno, M. C., Lambeth, S. P., \& Schapiro, S. J. (2009). Chimpanzees (Pan troglodytes) do not develop contingent reciprocity in an experimental task. Animal Cognition, 12, 587-597.

Brosnan, S. F., Wilson, B. J., \& Beran, M. J. (2012). Old World monkeys are more similar to humans than New World monkeys when playing a coordination game. Philosophical Transactions of the Royal Society B: Biological Sciences, 279, 1522-1530.

Brown, J. L. (1983). Cooperation - A biologist's dilemma. In J. Rosenblatt (Ed.), Advances in the study of behavior (pp. 1 -39). New York: Academic Press.

Bshary, R., \& Bergmüller, R. (2008). Distinguishing four fundamental approaches to the evolution of helping. Journal of Evolutionary Biology, 21, 405-420.

Claidière, N., Whiten, A., Mareno, M. C., Mxesser, E. J. E., Brosnan, S. F., Hopper, L. M., ....McGuigan, N. (2015). Selective and contagious prosocial resource donation in capuchin monkeys, chimpanzees and humans. Scientific Reports, 5, 7631.

Colman, A. D., Liebold, K. E., \& Boren, J. J. (1969). A method for studying altruism in monkeys. Psychological Record, 19, 401-405.

Crofoot, M. C., Rubenstein, D. I., Maiya, A. S., \& Berger-Wolf, T. Y. (2011). Aggression, grooming and group-level cooperation in white-faced capuchins (Cebus capucinus): Insights from social networks. American Journal of Primatology, 73, 821-833.

de Waal, F. B. M. (1997). Food transfers through mesh in brown capuchins. Journal of Comparative Psychology, 111, 370-378.

de Waal, F. B. M. (2000). Attitudinal reciprocity in food sharing among brown capuchin monkeys. Animal Behaviour, 60, 253-261.

de Waal, F. B. M., \& Berger, M. L. (2000). Payment for labour in monkeys. Nature, 404, 563-563.

de Waal, F. B. M., Leimgruber, K., \& Greenberg, A. R. (2008). Giving is self-rewarding for monkeys. Proceedings of the National Academy of Science, 105, 13685-13689.

de Waal, F. B. M., Luttrell, L. M., \& Canfield, M. E. (1993). Preliminary data on voluntary food sharing in brown capuchin monkeys. American Journal of Primatology, 29, 73-78. 
Di Bitetti, M. S. (1997). Evidence for an important social role of allogrooming in a platyrrhine primate. Animal Behaviour, 54, 199-211.

Drea, C. M., \& Wallen, K. (1999). Low-status monkeys "play dumb" when learning in mixed social groups. Proceedings of the National Academy of Sciences, 96, 12965-12969.

Dugatkin, L. A. (1991). Dynamics of the TIT FOR TAT strategy during predator inspection in the guppy (Poecilia reticulata). Behavioral Ecology and Sociobiology, 29, 127-132.

Dugatkin, L. A. (1997). Cooperation among animals: An evolutionary perspective, Oxford Series in Ecology and Evolution. New York: Oxford University Press.

Evans, T. A., Beran, M. J., Chan, B., Klein, E. D., \& Menzel, C. R. (2008). An efficient computerized testing method for the capuchin monkey (Cebus apella): Adaptation of the LRC-CTS to a socially housed nonhuman primate species. Behavior Research Methods, 40, 590-596.

Evans, T. A., Beran, M. J., Paglieri, F., \& Addessi, E. (2012). Delaying gratification for food and tokens in capuchin monkeys (Cebus apella) and chimpanzees (Pan troglodytes): When quantity is salient, symbolic stimuli do not improve performance. Animal Cognition, 15, 539-548.

Fragaszy, D. M., Visalberghi, E., \& Fedigan, L. M. (2004). The complete capuchin: The biology of the genus Cebus. Cambridge, UK: Cambridge University Press.

Fujita, K. (2009). Metamemory in tufted capuchin monkeys (Cebus apella). Animal Cognition, 12, $575-585$.

Gros-Luis, J., Perry, S., \& Manson, J. H. (2003). Violent coalitionary attacks and intraspecific killing in wild whitefaced capuhins monkeys (Cebus capucinus). Primates, 44, 341-346.

Jensen, K., Hare, B., Call, J., \& Tomasello, M. (2006). What's in it for me? Self-regard precludes altruism and spite in chimpanzees. Proceedings of the Royal Society of London B: Biological Sciences, 273, 1013-1021.

Lakshminarayanan, V. R. \& Santos, L. R. (2008). Capuchin monkeys are sensitive to others' welfare. Current Biology, 18, R999-R1000.

Leimgruber, K. L., Ward, A. F., Widness, J., Norton, M. I., Olson, K. R., Gray, K., \& Santos, L. R. (2014). Give what you get: Capuchin monkeys (Cebus apella) and 4-year-old children pay forward positive and negative outcomes to conspecifics. PLoS ONE, 9, e87035.

Manson, J. H., Navarrete, C. D., Silk, J. B., \& Perry, S. (2004). Time-matched grooming in female primates? New analyses from two species. Animal Behaviour, 67, 493-500.

Melis, A. P., Hare, B., \& Tomasello, M., (2008). Do chimpanzees reciprocate received favours? Animal Behaviour, 76, 951-962.

Michels, A. (1998). Sex differences in food acquisition and aggression in captive common marmosets (Callithrix jacchus). Primates, 39, 549-556.

Milinski, M. (1987). Tit for tat in sticklebacks and the evolution of cooperation. Nature, 325, 433-435.

Parrish, A. E., Brosnan, S. F., Wilson, B. J., \& Beran, M. J. (2014). Differential responding by rhesus monkeys (Macaca mulatta) and humans (Homo sapiens) to variable outcomes in the Assurance game. Animal Behavior and Cognition, 1, 215-229.

Perry, S., Manson, J. H., Dower, G., \& Wikberg, E. (2003). White-faced capuchins cooperate to rescue a groupmate from a Boa constrictor. Folia Primatologica, 74, 109-111.

Perry, S., \& Rose, L. (1994). Begging and transfer of coati meat by white-faced capuchin monkeys, Cebus capucinus. Primates, 35, 409-415.

Ramseyer, A., Pelé, M., Dufour, V., Chauvin, C., \& Thierry, B. (2006). Accepting loss: The temporal limits of reciprocity in brown capuchin monkeys. Proceedings of the Royal Society B: Biological Sciences, 273, 179184.

Rutte, C., \& Taborsky, M. (2008). The influence of social experience on cooperative behaviour of rats (Rattus norvegicus): Direct vs generalised reciprocity. Behavioral Ecology and Sociobiology, 62, 499-505.

Silk, J. B., Brosnan, S. F., Vonk, J., Henrich, J., Povinelli, D. J., Richardson, A. S., ...Schapiro, S. J. (2005). Chimpanzees are indifferent to the welfare of unrelated group members. Nature, 437, 1357-1359.

Suchak, M., \& de Waal, F. B. M. (2012). Monkeys benefit from reciprocity without the cognitive burden. Proceedings of the National Academy of Science, 109, 15191-15196.

Washburn, D. A., Hopkins, W. D., \& Rumbaugh, D. M. (1990). Effects of competition on video-task performance in monkeys. Journal of Comparative Psychology, 104, 115-121.

Wilkinson, G. S. (1984). Reciprocal food sharing in the vampire bat. Nature, 308, 181-184.

Wright, A. A. (1999). Visual list memory in capuchin monkeys (Cebus apella). Journal of Comparative Psychology, $113,74-80$. 